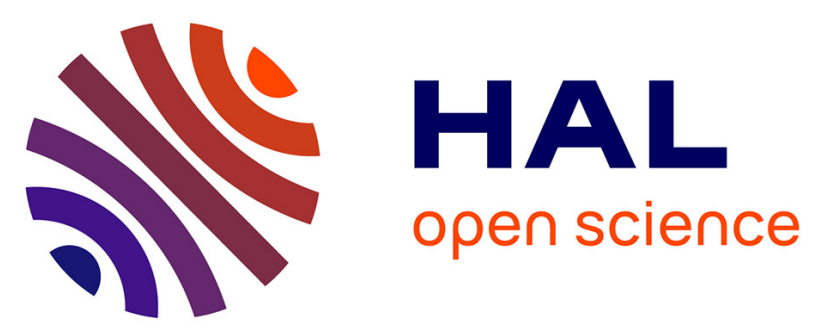

\title{
Motion Perspectives Integration in the Qualification of the Urban Spaces: Towards a 2D- and a 3D-Enrichment of the S-Partition Method
}

Thomas Leduc, Francis Miguet, Vincent Tourre

\section{- To cite this version:}

Thomas Leduc, Francis Miguet, Vincent Tourre. Motion Perspectives Integration in the Qualification of the Urban Spaces: Towards a 2D- and a 3D-Enrichment of the S-Partition Method. Barbara E.A. Piga; Rossella Salerno. Urban Design and Representation, 28, Springer International Publishing AG, pp.69-82, 2017, 978-3-319-51803-9. 10.1007/978-3-319-51804-6_6 . hal-01491469

\section{HAL Id: hal-01491469 \\ https://hal.science/hal-01491469}

Submitted on 16 Mar 2017

HAL is a multi-disciplinary open access archive for the deposit and dissemination of scientific research documents, whether they are published or not. The documents may come from teaching and research institutions in France or abroad, or from public or private research centers.
L'archive ouverte pluridisciplinaire HAL, est destinée au dépôt et à la diffusion de documents scientifiques de niveau recherche, publiés ou non, émanant des établissements d'enseignement et de recherche français ou étrangers, des laboratoires publics ou privés. 


\title{
Motion perspectives integration in the qualification of the urban spaces: towards a 2D- and a 3D-enrichment of the s-partition method
}

\author{
Leduc, Thomas (1); Miguet, Francis (1); and Tourre, Vincent (2) \\ (1) UMR CNRS AAU - Team CRENAU/ENSA Nantes - Univ. Bretagne Loire, France \\ (2) UMR CNRS AAU - Team CRENAU/Centrale Nantes - Univ. Bretagne Loire, France \\ thomas.leduc@crenau.archi.fr - +33240597599 \\ francis.miguet@crenau.archi.fr - +33240592122 \\ vincent.tourre@ec-nantes.fr - +33240371629
}

\begin{abstract}
The spatio-cognitive properties of urban surroundings must be taken into account in the design of urban spaces. However there are few methods and software tools to assess the visual properties and the spatial identity of places, and most of them are based on two-dimensional visibility analysis only and on a static analysis of surroundings. Our proposal aims to provide turnkey solutions that could increase the implementation of urban planning policies concerned with sensitive impacts of urban fabric renewal. The method presented in this chapter enhances the urban space partition proposed by Peponis et al. (1997) in order to qualify all pedestrian paths in the studied area taking into account the importance of changes in visual surroundings and the vertical landmarks. In our use case, the results of our method applied on two paths are compared with a 3D isovists analysis in order to clearly show the keypoints in the path. A software solution has been developed as a plugin of the well-known SketchUp computer-aided architectural design (CAAD) tool to be tested by students in the context of project teaching in School of Architecture.
\end{abstract}

Keywords: Convex partition; vertical landmark; 3D isovist 


\section{Introduction}

The design of urban spaces is a complex activity involving cognitive tasks related to numerous parameters. The projection into a spatial configuration to analyze the perception of surroundings and their influence onto the behavior of inhabitants is a difficult cognitive activity. It is possible to produce realistic urban environments in order to experiment them through numerical simulations (as virtual reality), but generating these realistic environments is a time consuming task with a significant cost. Another approach consists in analyzing the urban space with metrics and indicators, producing maps describing the qualities of spaces.

As these spaces are experimented, in the every-day life, while moving through them, it is necessary in introduce the people movements into the metrics. As we assume to focus on the pedestrian movement in this work, we have to qualify all the possible paths a pedestrian could walk along to go from one location to another. Therefore we propose a method to map the visual perception of urban morphology in order to analyze any of the potential paths into a predefined urban area. This perception of urban morphology is measured with tools based on visual appreciation. This method is synthetic, transposable and systemic in order to be applied on all urban areas. The mapping of the pedestrian visual perception is intended to identify and analyze the ambience potential which belongs to all the pathways, allowing the method to be independent of any given path beforehand.

Various methods to analyze open spaces or indoor spaces have already been developed in the state of the art. This includes, among others, the axial lines and axial maps of the space syntax from Hillier and Hanson (1984), the isovist fields developed by (Benedikt, 1979; Batty, 2001; Lonergan and Hedley, 2016), sky views and spherical analysis developed in particular by Teller (2003), the visibility graphs of Turner et al. (2001), and the convex partitioning of spaces such as the one proposed by (Hillier and Hanson, 1984; Hillier, 1996; Peponis et al., 1997).

The latter method (Peponis et al., 1997) proposes a partition of indoor spaces based on building shape, each facade is prolonged by a line until it finds another building blocking it: the s-line. Therefore, according to (ibid.), "Each time such an observer crosses an s-line, an entire surface either appears into the visual field or disappears outside it. For any two different s-spaces, there is at least one wall surface which is entirely visible from one but not from the other". Based on the principle that "surfaces and parts of surfaces may appear or disappear without crossing an s-line" (ibid.), the same authors propose also another space partition linking 
"extendible diagonals" (ibid.): the e-line. In this approach, the urban space is partitioned into a set of partition (s-partition or e-partition). "The e-partition represents a layout in terms of constituent convex spaces that are stable with respect to visual information" (Peponis and Bellal, 2010).

This is an efficient approach that can be computed easily but the analysis of the partitions is difficult, especially into old city centers, due to the combinatorial dimension (and its explosive growth!) of demarcation lines and convex spaces. The main interest of these partition-based methods is their intrinsic characteristic and their attempts to capture the structure of the involved architectural space through a dedicated discretization. Thus, they only rely on pure geometrical aspects of the urban fabric surroundings. The annoying counterpart of this intrinsic property is that these partition-based methods of urban spaces do not take into account the true visual perception of a moving pedestrian.

To overcome this drawback and to delineate iteratively the surroundings of a moving pedestrian (using the visual-based perceptive approach), another effective possibility consists in assessing the isovists field proposed by Benedikt (1979).

Whereas an isovist is the polygonal set of horizontal points in space that are visible from a given vantage point (up to the closest opaque facades surrounding the view point), the isovists field consists in some sort of scalar field which gives the ability to map the numerical (visual) properties in each vantage point. As noticed by (Peponis et al., 1997), "One implication of this is that isovist analysis, although easily applicable if we have reason to select some particular viewing points, cannot readily be automated or proceduralized to deal with an entire plan. It is always necessary to devise ways for sampling the set of points from which we will draw the isovist." Moreover, contrary to the convex partition approach, the parameters extracted from isovists do not clearly show the visual events that affects the visual perception. Therefore, the arising question can be set as: How to compute partitions in outdoor urban spaces showing homogeneous areas with the same visual elements, and how to highlight the importance of the visual events?

In order to map the ambience potential of urban open spaces, two different alternatives can be considered. The continuous approach, commonly referred as the fieldoriented open space analysis, consists in the spatial representation of any visual property (such as the surface of the corresponding isovist, or its perimeter as proposed by Benedikt (1979)) in each sampling position. Although precise and efficient, this field-oriented method does not exhibit easily the transitions between portions of space in terms of visual events. Indeed, in this conception of space, the void in between buildings is viewed as some continuous substrate (such as a 
"magnetic field") with fuzzy boundaries, and where sometimes some sub-portions of space overlap.

Conversely, in the discrete approach (such as the convex partitioning already mentioned), space is potentially dividable into identifiable partitions, defined a priori by clear and stable spatial limits. This approach provides the advantage to organize open space as a tessellation of discrete entities, which we can handle directly.

Our proposal is to extend the discrete approach (and more precisely the s-partition method proposed by Peponis et al. (1997)) so as to enhance it to handle urban open spaces in a more efficient way. This twofold adaptation consists in an integration of 3D saliences, and in a weighting of visual transitions clues according to the distances to corresponding generators.

\section{Outdoor convex partitions}

Our hypothesis is the following: by enhancing the convex partition method of Peponis et al. (1997) with a valuation of s-lines and the adding of vertical landmarks, we can produce areas with homogeneous visual elements, and we can quantify the importance of the transition between two s-partitions. We compute these partitions relying on an analytic approach based exclusively on the geometric shape of the buildings. The s-line shows a changing configuration of boundaries within the visual field, a visual event which is a discrete change in visual surroundings (ibid.): the appearance or disappearance of a façade in the field of view of the moving pedestrian produces the s-line. By weighting the s-lines and adding the v-lines (vertical lines), we can assess the difference between two isovists in two different partitions.

In space convex partitioning approach, the s-partition method splits the urban space by prolonging the facades footprints until encountering another facade that stop the cut. The space is then partitioned into several areas that share the same facades (visual obstacles) in their field of view. Crossing an s-line by moving from one s-partition to another (contiguous), means having one facade added or removed from the field of view. This move thus involves a visual event by the appearance or disappearance of the facade. But the difference between two spartitions are sometimes very slight because the visual event is quite unimportant, the facade producing the crossed s-line could be very far and/or very small. Therefore the full set of s-partition exhibits all the visual events without any contrast. Moreover the standard s-partition method is computed in 2D space, and does not take into account the height of buildings. Therefore some visual events caused by 
vertical landmarks or high buildings are not represented. Computing several 3D isovists on one s-partition clearly shows that these isovists could have a very different shape.

Our approach proposes two enhancements of the s-partition method: 1) we weight the s-line according to the distance to the corresponding facade (i.e. the facade that generates the given s-line), 2) we introduce the v-lines in the partition to take into account vertical landmarks. The benefits of these improvements are to qualify any given pathway and to quantify the importance of the changes of the visual events.

\section{Implementation}

The implementation of the outdoor convex partition method is achieved by weighting the s-line of the convex partition method and by introducing v-line. This section details the full process:

- Data preprocessing

- Building footprint generalization

- S-line generation

- V-line generation

- Partitioning

- S-line and v-line weighting

- Path definition and analysis

Data preprocessing

The spatial datasets we use are the ones provided by the IGN, the national French institute in charge of the management and updating of geodesic and leveling networks, aerial photographs, and geospatial data. The aforementioned datasets are part of the Large Scale Reference database (RGE®), a standardized and precise description of the French territory and the country's land use, and more precisely of its $\mathrm{BD}$ TOPO ${ }^{\circledR} 3 \mathrm{D}$ component, a 3D vector models of significant spatial features such as footprints of individual buildings, transport network, forest cover, etc. The needed pre-processing operations has been made using the Geospatial Data Abstraction Library GDAL/OGR (GDAL, 2016). Specifically the merging of the geospatial data and the clipping of the input data sources to some specified bounding box.

Generalize the Building footprints

As mentioned before, the aim is here to reduce as much as possible the complexity inherent to any real input dataset. Reducing this complexity while preserving topology means to minimize the number of useless nodes in buildings' exterior rings. This preprocessing consists in several operations such as the removing of the useless inner courtyards, the building footprints generalization using the Doug- 
las Peucker simplifier and a double buffer technique, the removing of the quasicollinear nodes, etc.

\section{S-line generation}

The s-line are generated using the standard convex partitioning method developed by Peponis et al. (1997). Each facade is prolonged by a line which divides the space into two partitions: one partition see the facade while the other not (fig. 1, left).

\section{$V$-line generation}

The same idea is used to generate the v-lines, the difference is that the generator is punctual and located on vertical landmark. The v-line can be seen as the border of the conic projection of the building on the ground, its center being the generator point (fig. 1, right). Four punctual generators at various heights are chosen on the vertical landmark (the Bretagne tower in the use-case presented hereafter), in order to generate four sets of v-lines delineating the portions of ground spaces in which these generators are visible or not. As these punctual generators may be arbitrarily positioned at gradual distances from the top of the vertical landmark, these several v-lines may show either the progressive, either the sudden apparition of the landmark in the field of view of the moving pedestrian.

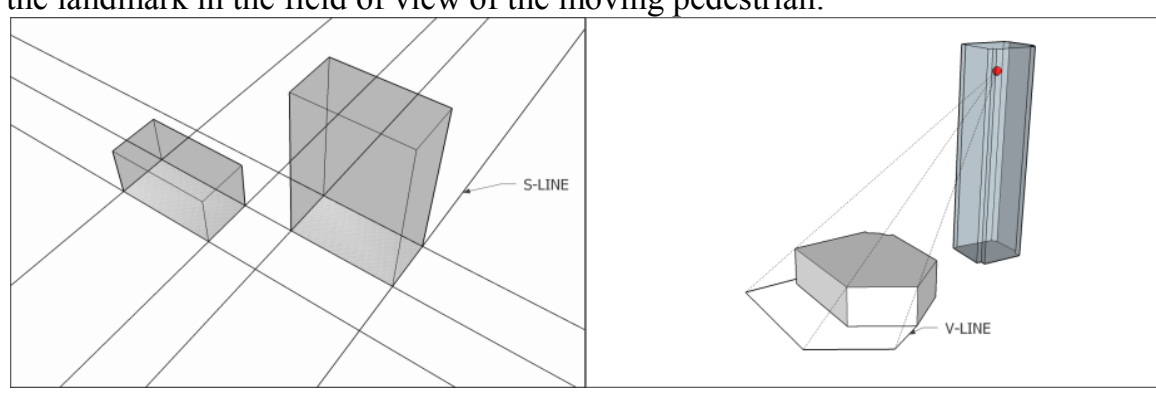

Fig. 1. s-lines (left) and v-lines (right) design drawings. Two different manners to subdivide the ground space in between the buildings.

\section{Partitioning}

The s-lines and v-lines are used to define the space partition. In each of the numerous items of this space partition, the visual properties are largely the same (both in terms of vertical saliences' visibility and in terms of breach or continuation of the visibility of surrounding facades).

\section{S-line and v-line scoring}

In order to distinguish the numerous items that result from this space partition, we have decided to score the contours according to their relative transition effect in terms of visual appreciation. Therefore, for each s-partition, the segments of sline contours are weighted according to the distance to the generating facade. In the same token, the various $\mathrm{v}$-lines contours are weighted according to the afore- 
mentioned punctual generators (lower is the elevation, greater is the visual impact).

\section{Path definition and analysis}

To demonstrate the viability of the method (an attempt to qualify a priori the urban fabric), several paths crossing a variety of urban viewscapes are defined. By systematic evaluation of the score of each crossed s- or v-line, a diagram is produced which renders the successive visual appreciations (emergence, vanishing) all along the path.

\section{Use case}

The chosen use case consists in a flat central district, mixing of quite homogeneous urban fabric with organic morphology, with some scattered and massive saliences. The use case area is $213,000 \mathrm{~m}^{2}$ wide containing 351 buildings footprints in $2 \mathrm{D}$ and represented by 4,717 nodes. A process in 5 steps allows to simplify the initial BD TOPO ${ }^{\circledR}$ data and then resulting in 62 footprints with 516 nodes in our use case. The figure 2 shows a subset of $87,000 \mathrm{~m}^{2}$ containing 36 footprints and 296 nodes: (1) simplification of the detailed footprint of Saint-Nicolas Church in order to reduce the number of generated s-lines (see fig. 2); (2) aggregation of the connected buildings in order to remove the common lines; (3) use of a dilatationerosion process to smooth the building footprints; (4) removing of the courtyards to keep only the public spaces; (5) removing of the quasi-linear nodes and Douglas-Peucker generalization.

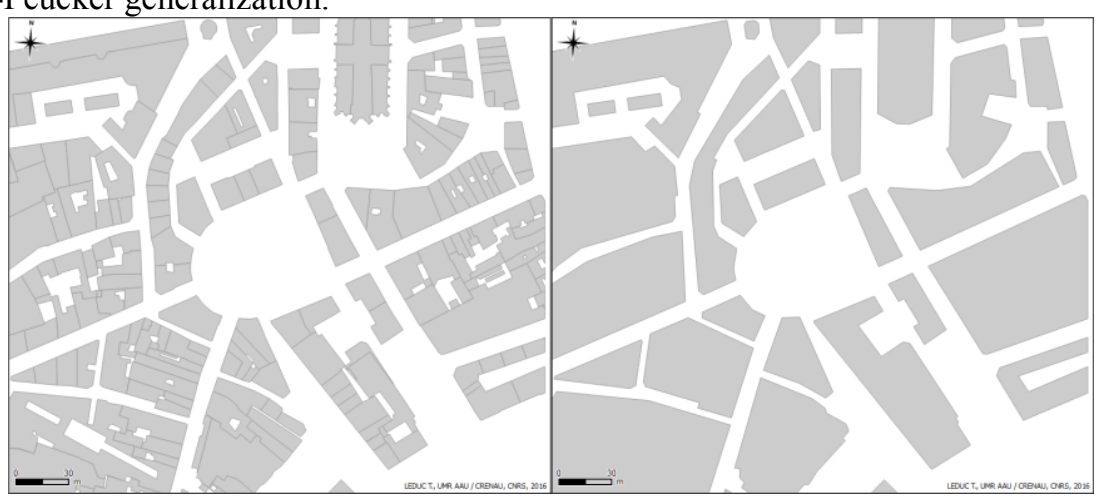

Fig. 2. The buildings' layer simplification process. The objective is to reduce the set of opaque edges able to generate visual cuts.

Starting from this $87,000 \mathrm{~m}^{2}$ subset $408 \mathrm{~s}$-line are generated (fig. 3, left). In order to enhance the legibility of resulting s-partitions, we choose a thematic cartographic representation with proportional symbols: the width of the s-lines is inversely to the distance from the generator. As shown in fig. 3 , right, from 0 to 5 
meters, the s-line is thick, from 5 meters to 26 meters, the s-line is medium, farther than 26 meters, the s-line is thin. This partition corresponds respectively to a strong, average and low influence in the field of view.

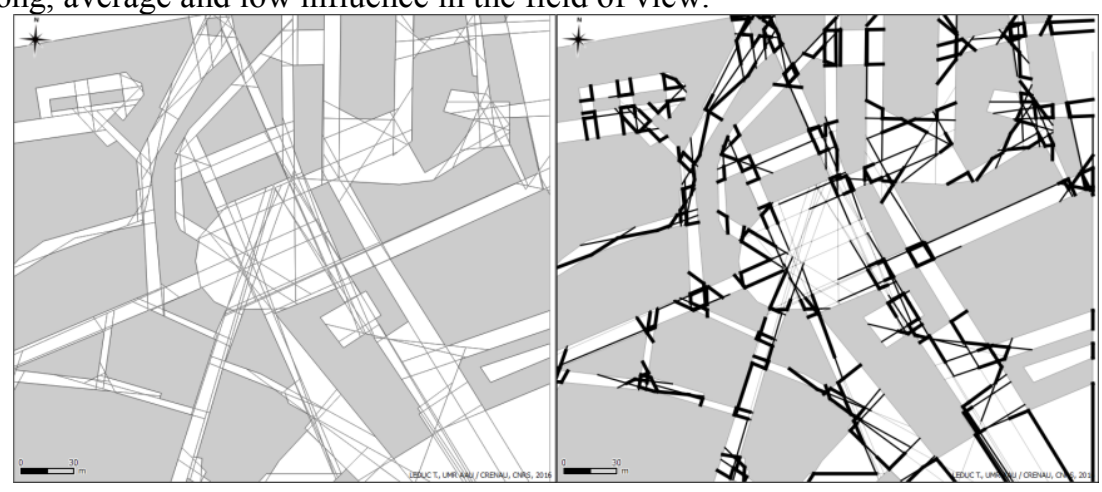

Fig. 3. The s-lines' layer weighting process. The objective is to enhance s-lines' readability.

Concerning the vertical landmarks, we only consider in this use case the Bretagne tower ${ }^{1}$ : A 37 -storeyed building of height 144 meters located about 200 meters north of the region of interest. The co-visibility areas presented in fig. 4 show four distinct thresholds: the v-line from the lightest grey to black shows the areas in which a point located at 12 meters, 22 meters, 32 meters, 42 meters respectively from the top and on the central axis of the Bretagne tower can be seen.

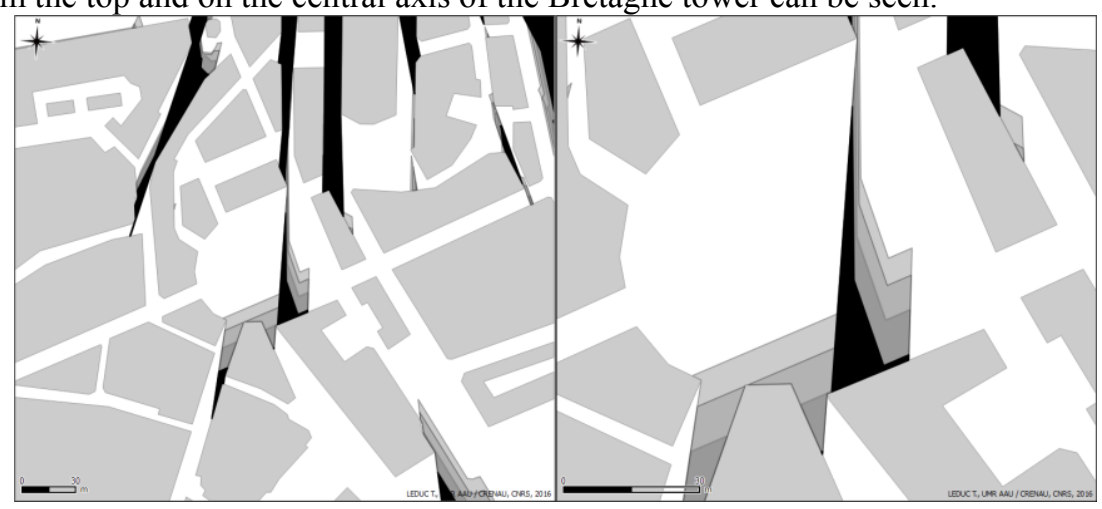

Fig. 4. The four set of v-lines with a zoom in on the Royale square (right). The black (resp. darkgrey, grey, and light-grey) polygons correspond to portions of ground space in which the punctual generator located at 42 meters (resp. 32 meters, 22 meters, and 12 meters) from top of the Bretagne tower is visible.

The fig. 5 shows two distinct pedestrian paths that help us to assess the enhancement of the convex partitions method that we developed. These paths start

${ }^{1}$ The tools developed with SketchUp allows to take into account other vertical landmarks as the bell tower of Saint-Nicolas church. 
from Commerce square located south in the region of interest and end up at the Bon Pasteur square. The first path: A, 320 meters long, bypass the Royale square on its west side and exhibits some obvious visual events in the field of view: outlet the Port au vin street on Fosse street (fig. 5, point A3), visibility of the Bretagne tower on the second half of Contrescarpe street (fig. 5, points A5 and A6). The second path: B, 280 meters long, crosses the Royale square from SW to NE (fig. 5 , points B1 to B4), goes straight into the Arche sèche street and turns into Échelle street (fig. 5, point B6) before taking the stairs to Bon Pasteur square (fig. 5, point B7).

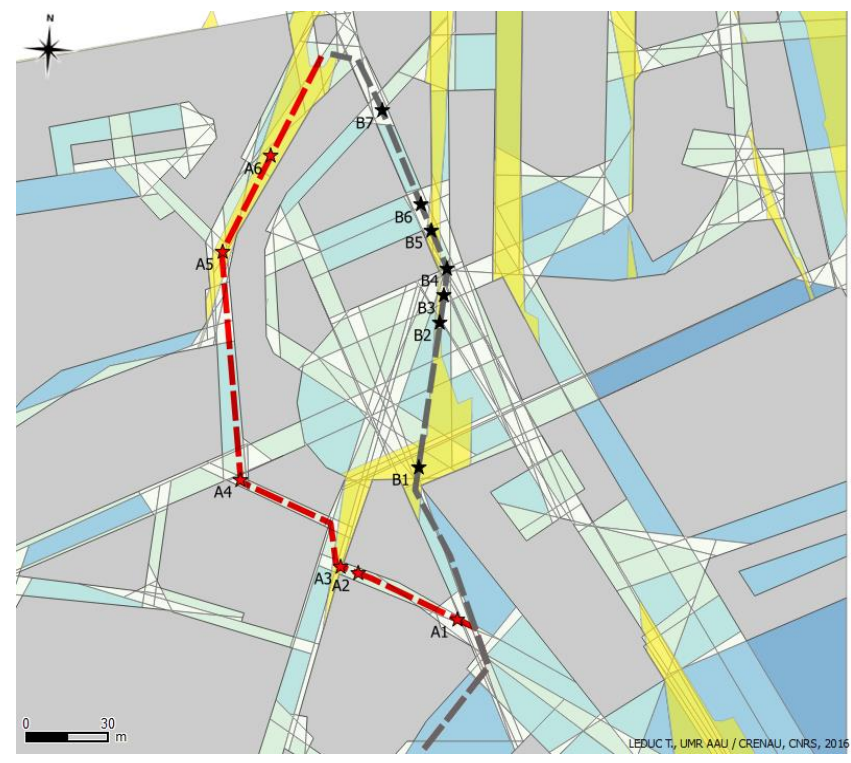

Fig. 5. The two studied urban paths with some emblematic positions. In the background map, spartitions and of yellow-colored areas of co-visibility with the Bretagne tower are presented.

The fig. 6 confirms with a fisheye picture, a fast $3 \mathrm{D}$ rendering in the $3 \mathrm{D}$ mockup, and the 3D isovist simulation the emergence of the Bretagne tower as a vertical landscape at point B5 in fig. 5. 


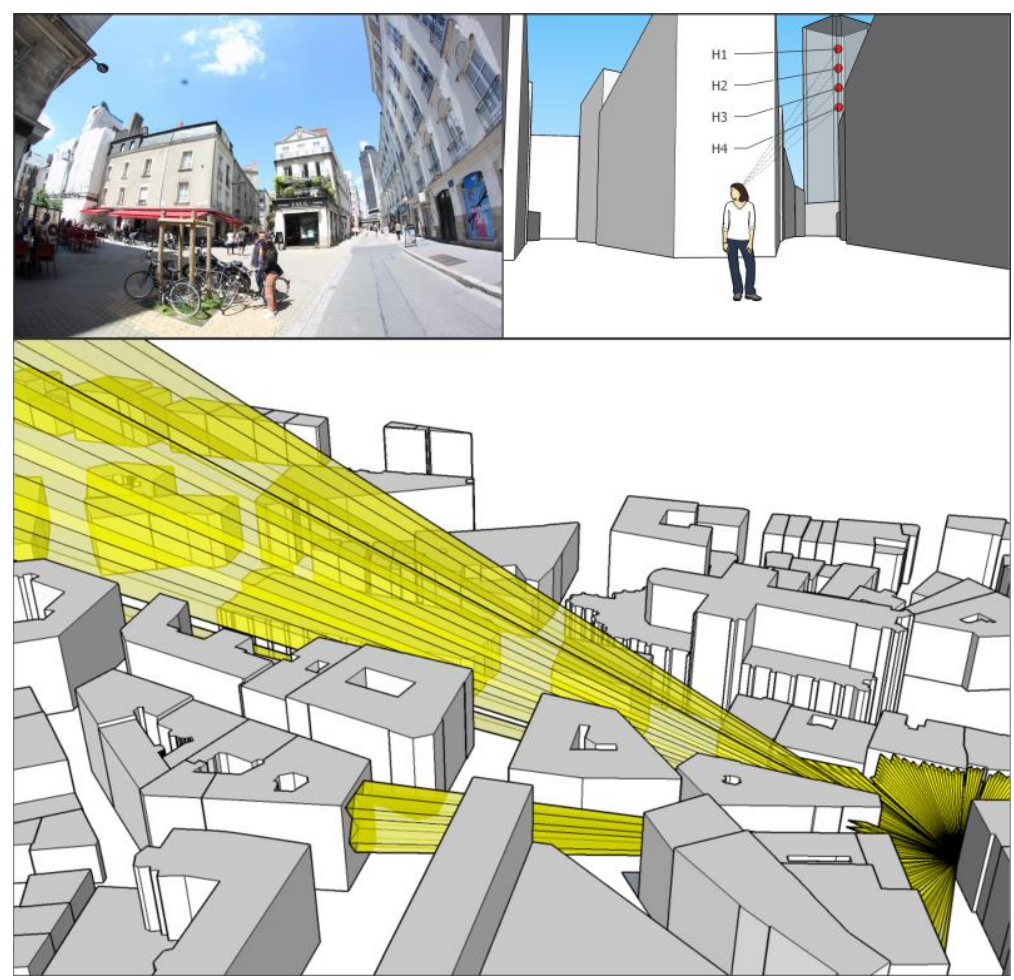

Fig. 6. The visual access of the Bretagne tower vertical landmark from the Arche Sèche Street (close to the point B5) is confirmed by all at once a fisheye photographic survey (top left), a perspective view from inside the 3D mock-up (top right), and a 3D isovist (bottom).

The color plate presented in fig. 7 summarizes the visual appreciations (the effects of the various visual properties) all along the path A. As one can notice at first glance, the second half of the Contrescarpe Street offers to the pedestrian a direct visual access to at least three of the punctual generators previously mentioned (the closest to the top of Bretagne tower). Regarding the s-lines' transitions, the kind of "frequency diagram" represented in this figure is less easy to encompass. Nevertheless, we may observe that the transition between the points A2 and A3 (from Port au Vin Street to the Fosse Street) is clearly underlined and similar to a tunnel exit. Lastly, after the A4 position, the crossing over Crébillon Street and the entrance of Contrescarpe Street causes a strong visual event.

Regarding the color plate presented in fig. 8, we obviously notice than the subareas linked to the v-lines are shorter but better distributed all along the path. The Bretagne tower is viewable regularly but each time for a small period. With reference to s-lines, one may note the lack of strong visual events between B1 and B3 positions (where the path $\mathrm{B}$ crosses the Royale square). More globally, comparing 
both frequency diagrams, the s-lines' transitions in path B seem less significant than in path $\mathrm{A}$.

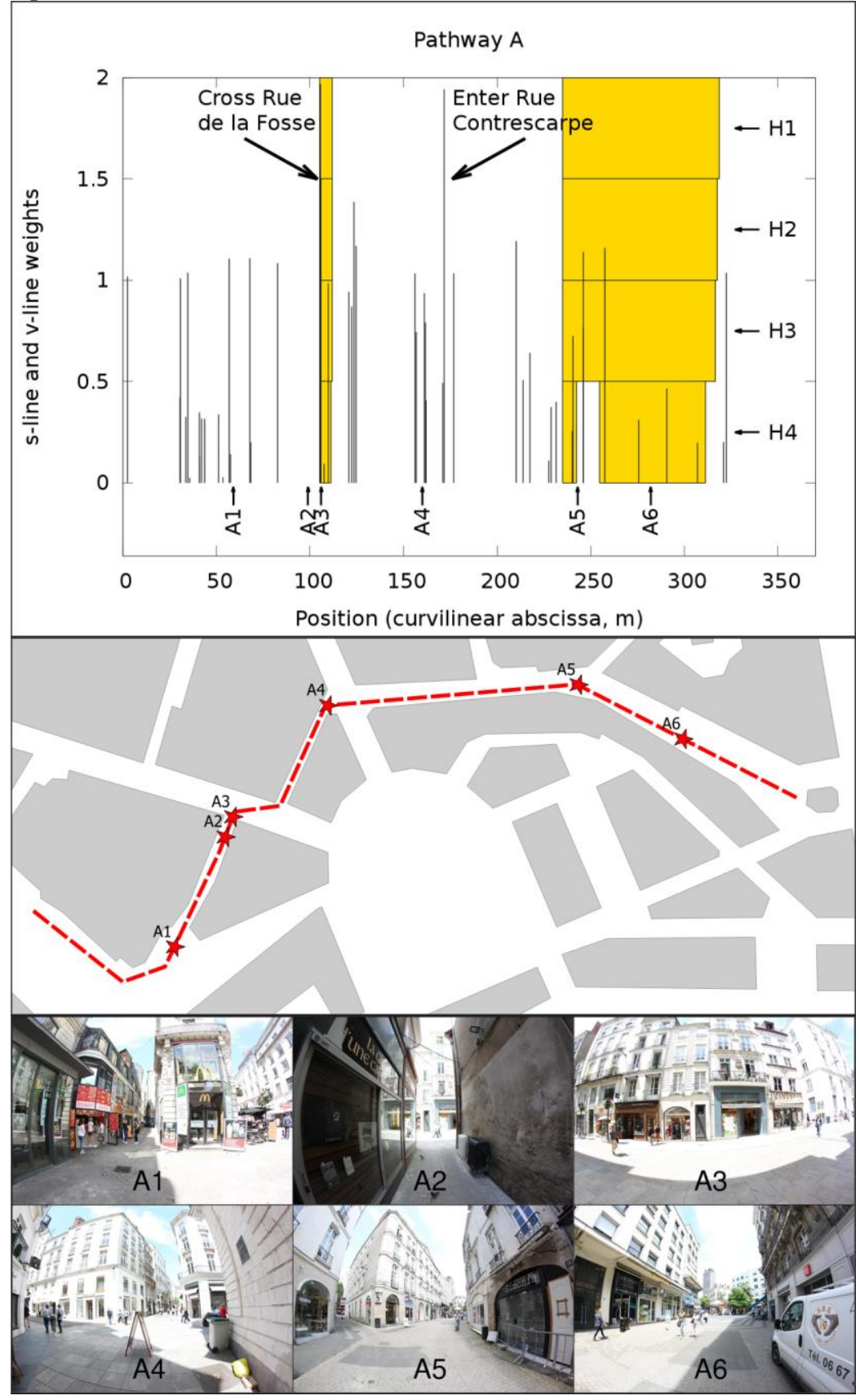

Fig. 7. A step-by-step description of path A in terms of visual transitions. 


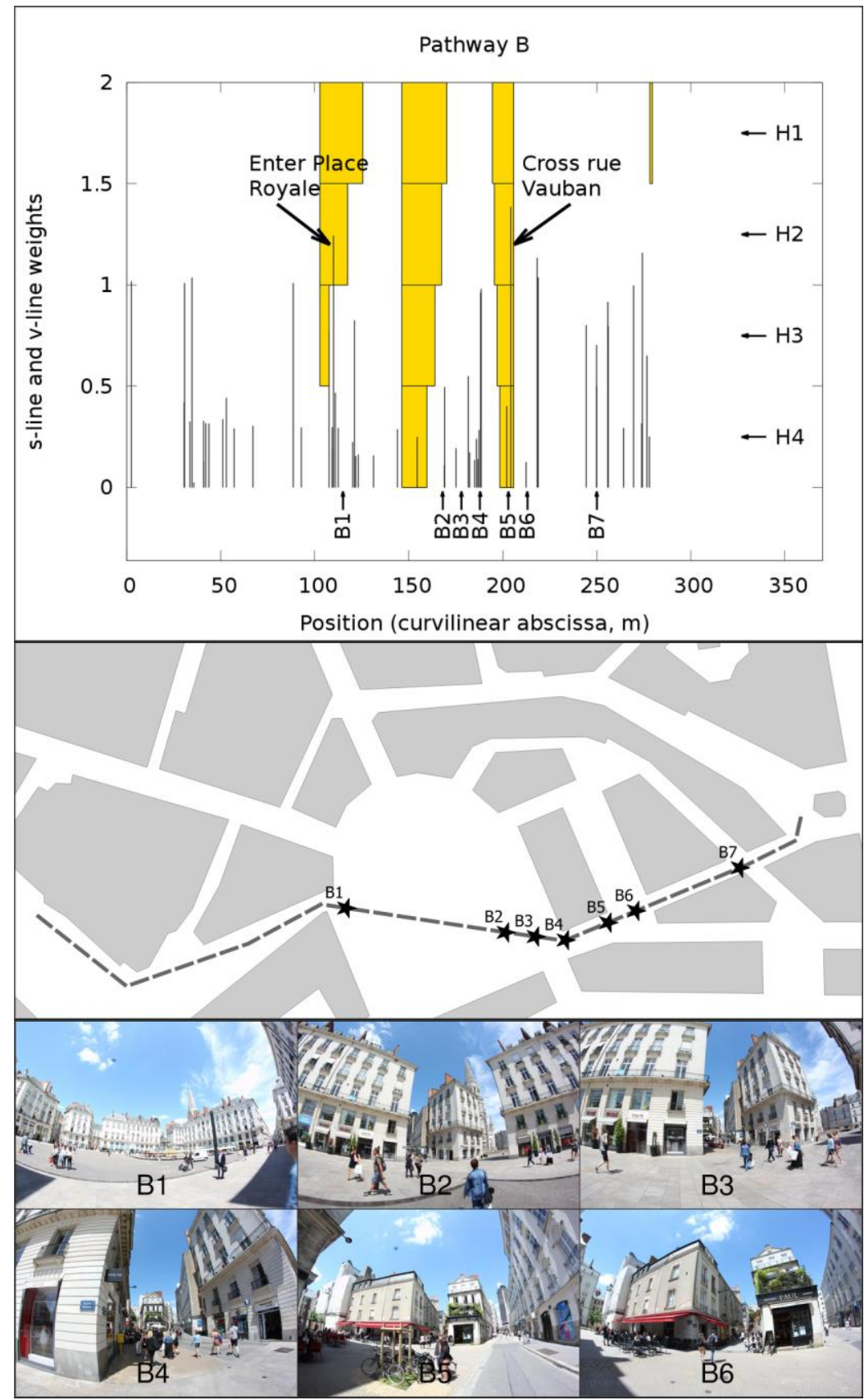

Fig. 8. A step-by-step description of path B in terms of visual transitions. 


\section{Discussion}

Fig. 9 shows an improved representation of visual events linked to s-line. From the points of the fig. 7 and 8 we have produced a curve with a natural smoothing spline operator. This arbitrary smoothing exhibits two phenomena related to spartitions: amplitude and frequency of visual events. The A path shows strong variations on long periods, and the path $\mathrm{B}$ shows small variations on short periods. Therefore concerning the horizontal visual events (s-lines), the A path could be described as varied while the B path can be described as more constant. And concerning the vertical visual events (v-lines), the A path is clearly less fluctuating than the B path.

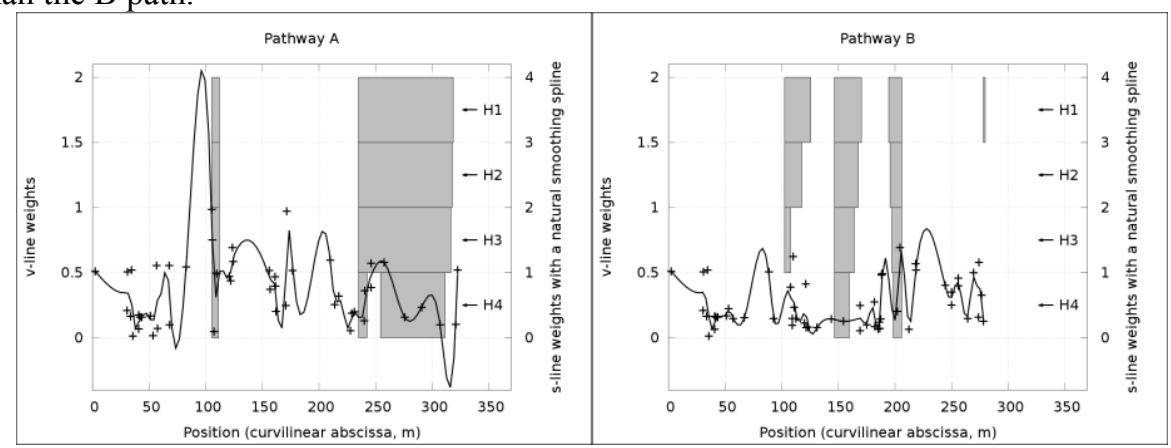

Fig. 9. Comparison of diagrams related to the visual properties of paths A and B.

This method could be used to show the discontinuities or visual events in the field of view and therefore shows also where we could find some important changes in $3 \mathrm{D}$ isovists. This assumption is limited by our approximation of vertical events: we only take into account the central axis of the vertical landmarks while they have an unneglectable width. This approximation allows more legible maps but at the cost of the precision of the results.

Concerning the e-lines, the question that arise is how the e-line and e-partitions could be weighted in order to be combined to the weighted s-line and v-line. In other words, how we could assess the importance of the visual events represented by e-line?

\section{Conclusion}

This study is a contribution to the open space convex partition solution proposed by Peponis et al. (1997). Our main goal is to enrich their pioneering proposal in two different manners. First one has to do with some sort of s-lines' weighting. Second one integrates landscape saliencies (from verticality point of 
view). Both extensions aim to put the emphasis on anthropocentric aspects, and to go beyond pure geometrical partition of open spaces.

Further work concerns the application of our method to the e-partitions in order to study the relative importance of e-lines compared to s-lines and v-lines. Secondly, the v-lines generation has to be done from any vertical landmark with some perceptive quality. And lastly we have to integrate the terrain model in the computation as the variations in the terrain could bring some new kind of convex partition of the space.

\section{References}

Batty, M. (2001). Exploring isovist fields: space and shape in architectural and urban morphology. Environment and Planning B: Planning and Design, 28(1), 123-150.

Benedikt, M. L. (1979). To take hold of space: isovists and isovist fields. Environment and Planning B: Planning and Design, 6(1), 47-65.

GDAL. (2016). GDAL - Geospatial Data Abstraction Library, Version 1.10.1. GDAL Development Team, Open Source Geospatial Foundation.

Hillier, B. (1996). Space is the machine. Press Syndicate of the University of Cambridge.

Hillier, B., Hanson, J. (1984). The Social Logic of Space. Cambridge: Cambridge University Press.

Lonergan, C., Hedley, N. (2016). Unpacking isovists: a framework for 3D spatial visibility analysis. Cartography and Geographic Information Science, 43(2), 87-102.

Peponis, J., Bellal, T. (2010). Fallingwater: the interplay between space and shape. Environment and Planning B: Planning and Design, 37(6), 982-1001.

Peponis, J., Wineman, J., Rashid, M., Hong Kim, S., Bafna, S. (1997). On the description of shape and spatial configuration inside buildings: convex partitions and their local properties. Environment and Planning B: Planning and Design, 24(5), 761-781.

Teller, J. (2003). A spherical metric for the field-oriented analysis of complex urban open spaces. Environment and Planning B: Planning and Design, 30(3), 339-356.

Turner, A., Doxa, M., O'Sullivan, D., Penn, A. (2001). From isovists to visibility graphs: a methodology for the analysis of architectural space. Environment and Planning B: Planning and Design, 28(1), 103-121. 